\title{
Focused Ion Beam Slicing of Cosmic Dust for Coordinated Structure-Isotope Studies
}

\author{
R.M. Stroud* \\ * Materials and Sensors Branch, Naval Research Laboratory, 4555 Overlook Avenue, Washington, \\ DC 20375
}

Coordinated, multi-instrument studies are essential for cosmic dust studies. The solar or presolar origin of extraterrestrial dust particles can only be determined from isotope measurements. The dust formation conditions and subsequent alteration processes can only be determined from structure and composition measurements. The combination of both set of measurements on the same dust grains enables examination of the processes of stellar evolution and dust formation, galactic chemical evolution, processing of dust in the interstellar medium and early solar system, and helps address the origin of our solar system. For example, coordinated studies of a rare subset of meteoritic aluminum oxide grains have demonstrated the origin of these grains to be in oxygen-rich Asymptotic Giant Branch stars pre-dating the Sun, that these grains are the first solid phase to condense around the AGB stars, and that they exhibit both crystalline and amorphous polytypes. These results confirm theoretical predictions of stellar dust condensation, and aid in the interpretation of infra-red spectra from the AGB stars [1]. Similar powerful conclusions have been drawn from other coordinated studies of presolar dust grains from meteorites [2] and interplanetary dust particles [3]. Such coordinated studies will be crucial for the analysis of the cometary and interstellar dust samples to be returned by the STARDUST mission.

The typical size of the cosmic dust grains is 0.1 to 10 microns. The largest ( $>\sim 3$ microns) of these are amenable to conventional embedding and microtoming techniques, and coordinated studies can be carried out on adjacent microtome sections $[2,3]$. The greater fraction of the grains is in the 0.1 to 3 micron range, which requires more sophisticated preparation techniques. We have developed focused ion beam techniques for sectioning individual sub-micron grains, before and/or after isotopic analysis, to facilitate further coordinated studies [1]. The ion slicing of the dust can be performed using a standard $\mathrm{Ga}^{+}$ion beam workstation and ex-situ lift-out tools. Greater flexibility in producing slices optimized for different analyses is possible with the combined electron beam-ion beam workstations and in-situ lift-out tools.

To date we have applied the ion slicing techniques to aluminum oxide, silicon carbide, and silicate presolar grains from meteorites, interplanetary dust particles, and meteoritic graphite grains with carbon isotopes consistent with formation in the primordial solar system. We will present results from coordinated TEM-SIMS studies of these grains, and discuss optimization of the ion slicing techniques for particular classes of cosmic dust, including aerogel-encased cometary dust to be returned by the STARDUST mission [4].

[1] R.M. Stroud et al., Science 305 (2004) 1455.

[2] F.J. Stadermann et al., Geochemica et Cosmochemica Acta 69 (2005) 177.

[3] L.P. Keller et al., Geochemica et Cosmochemica Acta 68 (2004) 2577.

[4] We gratefully acknowledge contribution of samples and isotopic analyses from Larry Nittler, Tom Bernatowicz, Peter Hoppe, Tim McCoy, and Ann Nguyen, and assistance with the dual-beam 
FIB from David Elbert. Financial support for this work has been provided by the Office of Naval Research and NASA.

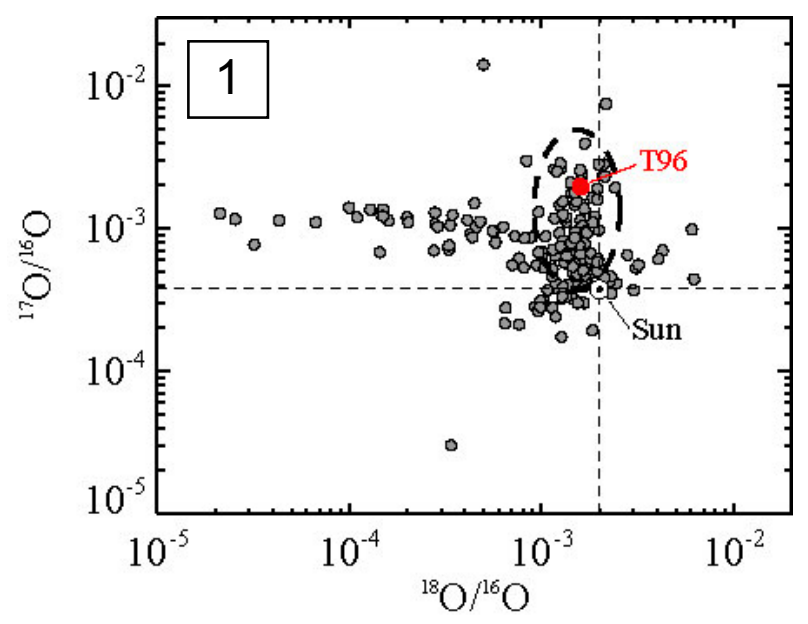

Figure 1. Oxygen isotope plot from presolar aluminum oxide grains. The dashed ellipse define the portion of grains with a origin in asymptotic giant branch stars. The label T96 indicates a grain from which a section was obtained using focused ion beam lift-out.
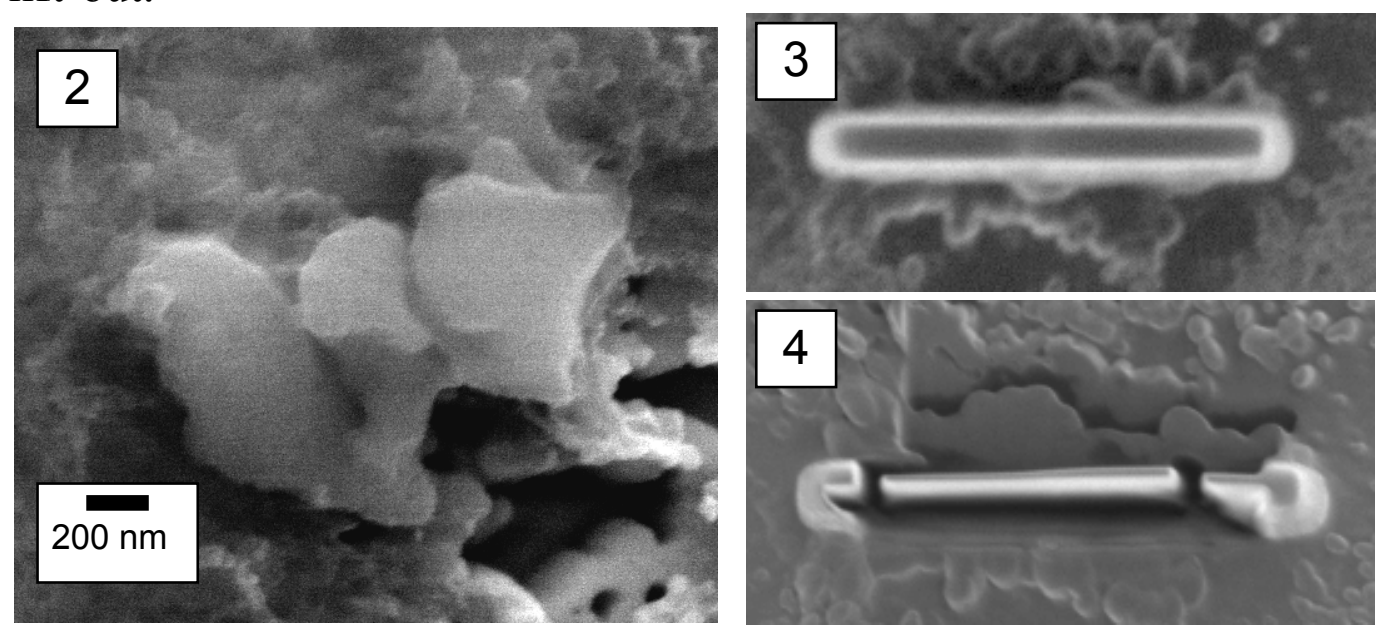

Figure 2. Secondary electron image of a 1-micron aluminum oxide grain after isotopic characterization demonstrating its presolar, stellar origin.

Figure 3. The grain from (2) after deposition of a 10-micron Pt mask using the gasinjection source in the focused ion beam workstation.

Figure 4. The electron-transparent membrane of the presolar grain and surrounding $\mathrm{Pt}$, ready for lift-out. 